\title{
PAINEL DE ESPECIALISTAS Dados de mundo real no processo de tomada
EXPERT PANEL de decisão: uma análise sob a perspectiva do sistema brasileiro de saúde suplementar
}

\author{
Real-world evidence in decision-making process: analysis \\ from the Brazilian private healthcare system perspective \\ André Ballalai', Nadja Nara Rehen de Souza², Janay Faria de Almeida da Silva², \\ Marina Moreira Suavinha ${ }^{4}$, Cláudia de Freitas Sousa ${ }^{5}$, Tassia Cristina Decimoni ${ }^{6}$, \\ Camila Rufino ${ }^{6}$ \\ DOI: 10.21115/JBES.v11.n3.p283-95
}

\section{Palavras-chave:}

tomada de decisões, dados de mundo real, reembolso de seguro de saúde, saúde suplementar, produtos biológicos

\section{Keywords:}

decision making, insurance, health, reimbursement, supplemental health, biological products

\section{RESUMO}

O debate sobre a necessidade de melhoria da utilização de recursos em saúde fez aumentar o interesse em evidências do mundo real (EMR) nos últimos anos. No sétimo congresso Latino-Americano da Sociedade Internacional de Farmacoeconomia e Pesquisa de Desfechos, diferentes palestrantes exploraram como o manejo desses dados pode auxiliar no processo de tomada de decisão. Quatro membros representando diferentes segmentos do Sistema de Saúde Suplementar (SSS) brasileiro foram convidados a participar de um painel de especialistas, a fim de entender suas percepções sobre o assunto. O nível de confiança na informação atualmente disponível variou entre 70\% e 90\% e os dados são utilizados na rotina de tomada de decisão. Considerando a utilização de evidências para prever decisões, os especialistas relatam não existir uma matriz institucional, mas que as informações existentes são utilizadas na construção de modelos preditivos por meio da criação de pacotes de serviços. A priorização da tomada de decisão é hoje essencialmente baseada em estimativas de custos. Apesar disso, são observadas diferentes situações em que dados de mundo real podem balizar esse processo. Existiu consenso de que uma mudança de paradigmas está ocorrendo e que esses processos representam um futuro plausível. O uso de EMR é de grande importância no processo de tomada de decisão na perspectiva do SSS e, acima de tudo, no suporte de modelos de saúde baseados em valor, sendo recomendado pela Agência Nacional de Saúde Suplementar como um pilar estratégico para a sustentabilidade do sistema.

\begin{abstract}
The debate about the needs for healthcare resource utilization improvement has been soaring during the last years. As a result, discussions on enhancements of the decision-making process through the leverage of real-world evidence (RWE) has also been fostered. In the 7th Latin American congress of the International Society for Pharmacoeconomics and Outcomes Research, different speakers explored how the management of these data support real-life healthcare decisions. Four members representing different segments from the Brazilian Supplementary Healthcare System (SHS) were invited to participate on an expert panel in order to understand their perceptions on this subject. The confidence level on available data ranges from $70 \%$ to $90 \%$ and information is used on decision-making routine. When the use of real-world evidence to predict decision was considered, panelists reported the absence of an institutional decision matrix, but that existing information is used to build predictive models through the creation of service packages. The prioritization of decision-making today is essentially based on the estimation of costs. However, different situations in which real-world data can
\end{abstract}

Recebido em: 19/11/2019 Aprovado para publicação em: 09/12/2019

1. IQVIA INC, USA

2. Sistema de Assistência à Saúde dos Servidores Públicos Estaduais (Planserv), Salvador, BA, Brasil

3. Unimed Curitiba, Curitiba, PR, Brasi

4. São Francisco, SP, Brasil

5. GEAP Saúde, Brasília, Brasil

6. Janssen-Cilag Farmacêutica, São Paulo, SP, Brasil

Instituição onde o trabalho foi executado: Janssen-Cilag Farmacêutica, São Paulo, SP, Brasil.

Congressos onde o estudo foi apresentado: Não foi apresentado até o momento.

Declaração: Este estudo foi financiado pela Janssen-Cilag Farmacêutica.

Autor correspondente: Camila Rufino, Janssen-Cilag Farmacêutica Ltda. Av. Pres. Juscelino Kubitschek, 2041. Complexo JK-

Torre B - 70 andar - N4. CEP: 04543-011 - São Paulo, SP, Brasil. Telefonte: +55 11 99149-4211. E-mail: crufino@its.jnj.com 
guide this process are observed. There was a consensus on a paradigm shift ongoing and that these processes represent a plausible future. The use of RWE is of great importance on decision-making process from the SHS perspective and, above all, in a support of value-based healthcare model which is recommended by Brazilian private agency (ANS) as the strategic pillar to system sustainability.

\section{Introdução}

O uso de evidências de ensaios clínicos randomizados (ECRs) está estabelecido como o padrão para determinar a eficácia e o perfil de segurança de intervenções em saúde. No entanto, já é amplamente aceito pela comunidade científica que ECRs limitam a população estudada em relação a diversos critérios, distanciando-se da realidade da prática clínica (Concato et al., 2000). Esses estudos contam com subpopulações homogêneas e seletas e, geralmente, são conduzidos sob circunstâncias ideais e controladas, o que pode não se estender necessariamente à população geral, heterogênea, com necessidades complexas de cuidados médicos, comorbidades múltiplas e/ou que faz uso concomitante de medicamentos não previstos no tratamento de uma doença (Katkade et al., 2018; Kim et al., 2018). Além disso, no contexto dos gestores de saúde, ainda há outras questões relevantes para a tomada de decisão como duração, custos e dificuldade de generalização dos achados. Isso ratifica a importância da busca por informações alternativas que auxiliem em processos, como na implementação de novas diretrizes para as operadoras de saúde (Franklin \& Schneeweiss, 2017; Sherman et al., 2016).

Nesse contexto, surge o interesse em dados de mundo real. A expressão "dados de mundo real" vem sendo amplamente utilizada por diferentes setores da assistência à saúde e está relacionada à informação derivada de diferentes fontes fora do contexto de ensaios clínicos tradicionais, o que pode incluir bases de dados eletrônicas, dados oriundos de contas médicas, de sistemas de prontuários eletrônicos, registros de produtos ou doenças, dados coletados por meio de dispositivos pessoais ou aplicativos de saúde, além daqueles gerados em estudos observacionais tanto retrospectivamente quanto prospectivamente. Portanto, são provenientes, na maioria das vezes, de fontes associadas ou utilizadas na prática clínica de rotina (Sherman et al., 2016).

Somado a isso, nos últimos anos, verifica-se uma tendência de órgãos regulatórios internacionais reconhecerem os estudos de mundo real como importantes repertórios de evidências científicas. Tem sido proposto o uso dessas evidências para a tomada de decisão em diferentes situações, tais como para aprovação primária (medicamentos novos), indicações secundárias (expansão da indicação ou da população), vias alternativas (uso de biomarcadores como desfechos clínicos, reivindicação de eficácia, ampliação da população) e segurança (avaliação pós-comercialização, resposta regulatória rápida a um alerta de segurança) (Franklin et al., 2019).

No entanto, apesar desse crescente interesse, há ainda divergência entre diferentes perfis de tomadores de decisão sobre a origem e o grau de confiabilidade das evidências de mundo real e sua utilidade (Makady et al., 2017). Somado a esse contexto, há também discussões legais a respeito de todo o espectro da proteção e privacidade dos dados em saúde. Por lei, determina-se que esse tipo de dado siga padrões mais rígidos que os dos demais, como é o caso do Health Insurance Portability and Accountability Act (HIPAA) nos Estados Unidos e da mais recente regulação de proteção de dados que vem sendo implementada na Europa (Annas, 2003; European Union, 2018).

Alguns autores têm se dedicado a entender como o processo de uso das evidências de mundo real está acontecendo. Em países da América do Norte, esse tipo de evidência tem sido utilizada especialmente para avaliação de segurança, manejo da utilização de tecnologias e análises de custo, porém, ainda em menor frequência, para a tomada de decisão sobre incorporação ou aprovação de novas tecnologias (Hampson et al., 2018; Husereau et al., 2019; Malone et al., 2018). No Oriente Médio, há ainda um entendimento de que o uso desses dados para a tomada de decisão é insuficiente, porém há um crescente interesse pelo tema (Akhras et al., 2019). Justo et al. (2019) apresentaram uma revisão sistemática com a finalidade de entender o uso de dados de mundo real na América Latina e relataram maior utilização para análise de segurança e geração de dados epidemiológicos e, em menor frequência, para a tomada de decisão sobre tecnologias em saúde ou negociações de preço (Justo et al., 2019).

Sob a perspectiva do Sistema de Saúde Suplementar (SSS), a importância do tema é crescente. Atualmente, o SSS atende a aproximadamente 47 milhões de brasileiros, o que corresponde a cerca de $24 \%$ da população do país. Destes, em torno de $70 \%$ dos beneficiários estão afiliados a planos de saúde na modalidade empresarial. Dessa forma, o número de beneficiários do sistema tem forte relação com as taxas de empregos formais (Agência Nacional de Saúde Suplementar (ANS), 2019a; Agência Nacional de Saúde Suplementar (ANS), 2019b). Dada sua amplitude de cobertura e relevância assistencial, entender o processo de tomada de decisão sob essa perspectiva se faz fundamental.

Considerando o contexto apresentado, especialistas no tema se reuniram durante o Congresso Latino-Americano da Sociedade Internacional de Farmacoeconomia e Pesquisa de Desfechos (International Society for Pharmacoeconomics and Outcomes Research; ISPOR) para discutir a importância, barreiras e oportunidades para uso de dados de mundo real na tomada de decisão.

Este trabalho tem a finalidade sumarizar as tendências apresentadas neste congresso e capturar a percepção dos gestores sobre o tema no Brasil. 


\section{Métodos}

No período entre 12 e 14 de setembro de 2019, ocorreu a sétima edição Latino-Americana do congresso da ISPOR. Esse evento ocorre com frequência bienal e é o principal canal de disseminação do estudo da farmacoeconomia na América Latina.

A primeira plenária intitulada "The role of data supporting an effective decision-making process" teve por objetivo explorar como o manejo desses dados pode influenciar as decisões em saúde e a alocação em recursos na América Latina. A discussão contou com a participação de cinco palestrantes:

- Manuel Antonio Espinoza, MD MSc PhD, Pontificia Universidad Católica, Santiago, Chile (moderador).

- Rafael Alfonso-Cristancho, MD, PhD, MSc, GlaxoSmithKline plc., Estados Unidos.

- Edson Amaro, MD, PhD, Hospital Israelita Albert Einstein, Brasil.

- William H Crown, PhD, OptumLabs, MA, Estados Unidos.

- Oscar Espinosa, MSc, Institute of Technology Assessment in Health, Bogota, Colômbia.

- Lucinda Orsini, ISPOR, Estados Unidos.

Os palestrantes apresentavam perfis distintos, sendo provenientes da academia, indústria farmacêutica, hospitais (prestador de serviço), consultorias especializadas na análise e manejo de informações, institutos de avaliação de tecnologia em saúde e membros da ISPOR, possibilitando um debate com diferentes visões sobre o tema.

\section{Perspectiva de tomadores de decisão do Sistema de Saúde Suplementar brasileiro}

A fim de entender a importância do uso de dados de mundo real para o processo de tomada de decisão sob a perspectiva do SSS brasileiro, quatro representantes dos diferentes modelos assistenciais (autogestão, autarquia, cooperativa e me- dicina de grupo) desse sistema foram convidados a assistir a plenária apresentada durante o congresso da ISPOR América Latina e, posteriormente, debater sobre o tema e o seu uso na prática diária de tomada de decisão. O formato adotado para a discussão foi um painel de especialistas com moderação de um consultor especialista no tema.

Foram desenvolvidas seis perguntas (Quadro 1) a partir dos direcionamentos discutidos pelos palestrantes durante a plenária do congresso, as quais foram debatidas pelos gestores brasileiros a fim de identificar a percepção em relação aos temas, barreiras e oportunidades, além de tendências sobre o assunto.

\section{Análise complementar}

Com propósito de ampliar o estudo e validar as percepções capturadas inicialmente, uma análise suplementar foi conduzida por consultor especialista no assunto, que participou da discussão com os gestores brasileiros. A pesquisa foi enviada a 20 outros gestores de operadoras de saúde no período de 15/09/2019 a 30/10/2019. Foi utilizado um questionário online (Anexo 1), composto por quinze perguntas, todas de múltipla escolha e/ou de ranking de atributos por meio de três dimensões (Quadro 2). As análises e os resultados desse estudo foram utilizados de maneira agregada exclusivamente para o desenvolvimento dessa publicação e em caráter de confidencialidade.

\section{Resultados}

\section{Discussão 1 - Percepções atuais e mudança de paradigmas}

A plenária do congresso foi iniciada com uma discussão sobre a evolução dos dados e dos métodos para a geração de evidência de mundo real, destacando o uso de tecnologias como inteligência artificial e machine learning. Na percepção dos gestores brasileiros, esses são temas importantes, mas ainda distantes da realidade. Os desafios atuais permanecem centrados no acesso, integração ou uso de sistemas computadorizados.

Quadro 1. Perguntas norteadoras da discussão com gestores brasileiros

\begin{tabular}{ll}
\hline Pergunta 1 & $\begin{array}{l}\text { O primeiro palestrante apresentou tendências que suportarão a geração de dados de mundo real. Qual é } \\
\text { sua visão sobre isso? }\end{array}$ \\
\hline Pergunta 2 & $\begin{array}{l}\text { O segundo palestrante apresentou desafios relacionados aos estudos de mundo real. Defendeu que hoje } \\
\text { existem muitos dados, muitas informações em potencial, mas questionou como gerar informações válidas. } \\
\text { Qual é a sua opinião sobre isso? }\end{array}$ \\
\hline Pergunta 3 & $\begin{array}{l}\text { O terceiro palestrante estruturou cada tipo de fonte de evidências e definiu a melhor aplicabilidade da } \\
\text { informação para a tomada de decisão. Qual sua opinião sobre essa forma de avaliação? }\end{array}$ \\
\hline Pergunta 4 & $\begin{array}{l}\text { O quarto palestrante apresentou modelos preditivos que contribuem para antecipar desfechos como nas } \\
\text { decisões de uso de recurso apoiando o direcionamento de atividades e orientando a tomada de decisão no } \\
\text { hospital. Qual é sua opinião sobre isso? }\end{array}$ \\
\hline Pergunta 5 & $\begin{array}{l}\text { O quinto palestrante desafiou o atual modelo de avaliação de dados trazendo uma provocação para } \\
\text { tomada de decisão em menor tempo com a maior assertividade possíveis. Para isso, apresentou como } \\
\text { possibilidade o uso de dados de mundo real e questionou como a indústria farmacêutica pode contribuir } \\
\text { nesse processo. Qual é sua opinião sobre isso? }\end{array}$ \\
\hline Pergunta 6 & $\begin{array}{l}\text { Considerando a discussão sobre utilização de dados de vida real no processo de decisão, como você } \\
\text { aplicaria essa abordagem dentro do tema de imunobiológicos? }\end{array}$ \\
\hline
\end{tabular}


ANEXO I - Questionário análise adicional

\section{Utilização de evidências de mundo real no sistema de saúde suplementar brasileiro}

Identificação

1. Você concorda com os termos acima ao fazer parte do estudo?

Caso tenha interesse em receber o resultado completo do estudo, por favor deixe o seu contato (e-mail preferencialmente) para que possamos entrar em contato futuramente enviando a análise completa do estudo.

Suas informações e contato serão mantidos em caráter sigiloso e de acesso restrito e exclusivo ao pesquisador que está liderando o estudo.

2. Dados para envio do resultado do estudo (opcional):

Nome (opcional)

Empresa (opcional)

E-mail (opcional)

Número de telefone (opcional)

3. Qual a sua função atual dentro da operadora de saúde?

$\square$ Auditor/Consultor de contas médicas (terceiro/independente)

$\square$ Auditor/Consultor de contas médicas (interno/CLT)

$\square$ Gestor de auditoria médica/contas de autocusto

$\square$ Gestor clínico/médico

$\square$ Gestor de operações/regulação

$\square$ Gestor de relacionamento com clientes/comercial

$\square$ Gestor de relacionamento com clientes/comercial

\section{Dimensão 1 - Informações descritivas da operadora de saúde}

As informações desta seção inicial serão utilizadas exclusivamente para fins de análise de subgrupos quando relevantes.

1. Qual é a classificação da operadora de saúde segundo à ANS?

$\square$ Seguradora

$\square$ Medicina de grupo

$\square$ Cooperativa médica

$\square$ Autogestão

$\square$ Filantropia

$\square$ Autarquia (não regulada pela ANS)

2. Qual o tamanho atual da operadora com base no número de afiliados ativos?
$\square 0$ a 9.999
$\square 10.000$ a 49.999
$\square 50.000$ a 149.000
$\square 150.000$ a 499.999
$\square 500.000$ a 1.499 .999
$\square 2.000 .000$ ou mais

3. A sua operadora tem rede própria e/ou estrutura verticalizada?

$\square$ Sim, altamente verticalizada

$\square$ Sim, parcialmente verticalizada

$\square$ Não

\section{Dimensão 2 - Uso atual/futuro de evidências de mundo real}

Nesta seção vamos abordar a utilização dos dados de mundo real da sua operadora no processo de tomada de decisão.

Responda às perguntas considerando a utilização das informações/dados internos da operadora que atualmente você é parte.

1. Você utilizou dados de mundo real em análises internas ou para o suporte da tomada de decisões incluindo cobertura, autorizações ou atualização de formulários/protocolos/diretrizes nos últimos 3 anos?
$\square \operatorname{Sim}$
$\square$ Não 


\section{Utilização de evidências de mundo real no sistema de saúde suplementar brasileiro}

2. Dos processos descritos abaixo, quais destes você já utilizou dados de mundo real? Assinale mais de uma alternativa em caso de múltiplas opções

$\square$ Levantamento de custos/utilização de recursos para análises financeiras/gerências internas.

$\square$ Levantamento de custos/utilização de recursos para suportar negociações com provedores, fabricantes e/ou distribuidores

$\square$ Avaliação de utilização de recurso e desfechos durante o processo de autorização de cobertura

$\square$ Estudos de economia da saúde para o desenvolvimento e atualização de formulários, protocolos e diretrizes internas

$\square$ Estudos de economia da saúde para a análise de inclusão de procedimentos/medicamentos não listados no ROL da ANS

$\square$ Estudos de mundo real e economia da saúde para fins acadêmicos (e.g. publicações, simpósios e congressos)

$\square$ Análises estatísticas preditivas e/ou utilizando metodologias de inteligência artificial para o suporte na tomada de decisão

3. Atualmente, você utiliza dados de mundo real durante o processo de avaliação/autorização de cobertura?

$\square$ Nunca utilizel

$\square$ Poucas vezes e/ou casos em específicos

$\square$ Consideravelmente, em casos que julgo necessário como informação de suporte

$\square$ Sempre utilizo dados de mundo real durante a análise de autorização de cobertura

4. Atualmente, você utiliza dados de mundo real para definir/atualizar formulários, protocolos e diretrizes?

$\square$ Nunca utilizei

$\square$ Poucas vezes e/ou em formulários, protocolos, e diretrizes específicas

$\square$ Consideravelmente, em casos que julgo necessário como suporte

$\square$ Sempre utilizo dados de mundo real da minha operadora para desenvolver/atualizar formulários, protocolos ou diretrizes

5. Qual é o seu grau de confiabilidade na utilização dos dados de mundo real da sua operadora durante o processo de tomada de decisão?

$\square$ Não confio: não me sinto confortável em usar evidências de mundo real para tomar decisões

$\square$ Pouco confiável: utilizo evidências de mundo real em ocasiões específicas quando outras opções não estão disponíveis

$\square$ Confiável: confio nos dados de mundo real disponíveis, porém utilizo como uma ferramenta de suporte a outras fontes principais como dados clínicos e estudos randomizados

$\square$ Muito confiável: utilizo dados de mundo real sempre que disponíveis, e em algumas vezes utilizo esses dados como fonte principal para a tomada de decisão

6. Quais os principais motivos influenciam no grau de confiabilidade dos dados de mundo real da sua operadora? Ordene as opções de forma que a primeira seja o fator mais impactante e a última, o menos impactante

$\square$ Tamanho da amostra disponível comparada com o universo da operadora

$\square$ Grau de completude dos dados (e.g. campos relevantes não preenchidos e/ou com baixa taxa de preenchimento)

$\square$ Problemas no processo de input dos dados por parte do profissional de saúde envolvidos no processo (e.g. erros de digitação, preenchimento imparcial dos dados, campos com valores genéricos etc.)

$\square$ Rastreabilidade do histórico longitudinal do paciente é pouco representativo (tempo de jornada disponível dentro da base de dados)

\section{Dimensão 3 - Disponibilidade e utilização de evidências de mundo real dentro da operadora}

Nesta seção, vamos abordar temas relacionados à qualificação dos dados de mundo real, seu grau de disponibilidade, assim como os processos internos para a utilização desses dados

1. Qual é o grau de disponibilidade de dados de mundo real dentro da sua operadora?

$\square$ Não está disponível/Acesso restrito

$\square$ Pouco disponível/Acesso restrito: existem dados, porém com acesso restrito/difícil acesso (e.g. prontuários em papel, formato eletrônico muito antigos, não existe interface para extrair os dados do sistema)

$\square$ Pouco disponível/Não estruturado: os dados estão disponíveis em bancos de dados distintos e sem nenhuma estruturação e/ou padronização e requer autorização prévia para sua utilização

$\square$ Parcialmente disponível/Não estruturado: uma parcela dos dados está disponível para utilização, porém exige esforço considerável de estruturação, organização e limpeza previamente a sua utilização

$\square$ Disponível/Pouco estruturado: a maioria dos dados está disponível, porém apenas uma parcela de maneira estruturada, o que requer trabalho extensivo de organização e limpeza dos dados antes da análise gerencial

$\square$ Disponível/Estruturado: dados estão estruturados, porém sem integração com sistema de análise (e.g. bases de dados precisam ser carregadas manualmente em ferramentas de análise) 
Quadro 2. Dimensões analisadas por meio do questionário enviado aos gestores incluídos na análise

\begin{tabular}{lll}
\hline Dimensão 1 & $\begin{array}{l}\text { Informações descritivas da } \\
\text { operadora de saúde }\end{array}$ & Três questões \\
\hline Dimensão 2 & $\begin{array}{l}\text { Uso atual/futuro de } \\
\text { evidências de mundo real }\end{array}$ & Seis questões \\
\hline Dimensão 3 & $\begin{array}{l}\text { Disponibilidade e utilização } \\
\text { de evidências de mundo } \\
\text { real dentro da operadora }\end{array}$ & \\
\hline
\end{tabular}

Sobre o tema, as opiniões foram divergentes, variando de acordo com o tipo de assistência à saúde em que o gestor lida na sua prática. Considerando a perspectiva de gestores de autarquia e medicina de grupo, existe atualmente uma dificuldade de acesso ao dado por diferentes razões como a legislação vigente, a inexistência de bases de dados integradas e gestão dos dados por serviços terceirizados, porém, houve tentativas anteriores de implementação do uso de sistemas computadorizados. Já sob a perspectiva de sistemas verticalizados e de autogestão, existe planejamento de implementação atual ou futura do uso de tecnologias para geração de dados. Apesar das divergências, houve consenso sobre a importância da geração de informação de maneira sistematizada para a tomada de decisão.

Nessa perspectiva, houve consenso sobre a necessidade de mudança de cultura para que o uso de tecnologia de informação e a construção de um conjunto de dados para a tomada de decisão possam ser implementados nos diferentes modelos de assistência. Alguns estão já em processo de estruturação e outros ainda não, porém, a mudança de paradigma no processo de produção de informação é um consenso. Além disso, ideias de inovação são bem vistas e estimuladas, especialmente no modelo verticalizado.

\section{Discussão 2 - Confiança nos dados disponíveis para a tomada de decisão}

No segundo momento da plenária do congresso, discutiu-se que, com a crescente disponibilidade de informações, há uma maior preocupação com a qualidade dos dados. Diferentes obstáculos podem ser encontrados quando se deseja entender o conjunto de informações, como a infraestrutura e a tecnologia disponíveis, motivos legais como proteção à informação, ausência de pessoal capaz para trabalhar o dado, ou ausência de possibilidades metodológicas para coleta de maneira sistematizada.

Considerando os dados atualmente disponíveis para a tomada de decisão, os gestores brasileiros foram convidados a refletir sobre o grau de confiança nessas informações. O nível de confiança no dado atualmente disponível varia de $70 \%$ a 90\% entre os participantes do painel. Apesar de não haver confiança absoluta, em geral, a informação é considerada para a tomada de decisão. Todos os dados atualmente disponíveis passam por processo de auditoria e, por isso, mes- mo quando algum erro na informação é encontrado, estes são utilizados para ajuste de condutas ou processos internos. A ausência de confiança na informação disponível, mesmo sendo reduzida (variando de $10 \%$ a $30 \%$ ), está geralmente relacionada a dados incompletos ou insuficientes.

\section{Discussão 3 - Tomada de decisão propriamente dita com base em dados de mundo real}

A informação atualmente disponível é utilizada para o processo de tomada de decisão, mesmo não existindo uma completa confiança. Esses dados são utilizados para diferentes tipos de decisões, tais como definição de investimentos, de protocolos clínicos e possibilidade de verticalização do cuidado. Em alguns casos, a disponibilidade de informações do histórico do paciente possibilita a tomada de decisão que considere não somente a relação custo-efetividade, mas o entendimento de cada caso de maneira individual, como citado pela representante de uma autogestão. Além disso, a disponibilidade de informação permite conhecer a realidade dos beneficiários da operadora e possibilita uma posterior melhor alocação de recursos, mesmo quando o orçamento é reduzido.

A priorização da tomada de decisão é, em geral, baseada em estimativas de custos. Apesar disso, foram observadas diferentes situações em que os dados de mundo real podem balizar esse processo. Por exemplo, com a finalidade de evitar pagamentos mediante judicialização, as operadoras incorporam tecnologias não obrigatórias e acompanham a sua utilização por meio de dados de mundo real. Isso permite a avaliação em longo prazo sobre a necessidade de manutenção de diversas incorporações.

Considerando diferentes desfechos para a tomada de decisão, além do custo do medicamento propriamente dito, podem ser consideradas características como comodidade posológica e a efetividade do medicamento. A decisão pela cobertura de determinada tecnologia pode ainda ser tomada por uma equipe de especialistas que avaliam a necessidade de cada caso.

Mudanças nos modelos de tomada de decisão vêm sendo propostas, inclusive entre os gestores participantes do painel, possibilitando a observação de outros desfechos que não aqueles usualmente utilizados em ensaios clínicos. Dessa forma, foi possível verificar o fenômeno de transição de modelos de negociação financeiros majoritariamente baseados nos modelos de reembolso "fee for service" para estratégias mais modernas de negociação baseadas em valor (Figura 1), assim como uma tendência na maior adoção de acordos de risk-sharing - movimento que tem alta correlação com a disponibilidade de dados de mundo real, uma vez que algumas das estratégias são fundamentadas na mensuração dos desfechos de mundo real.

Atualmente, alterações nas estratégias de tratamento ocorrem com base em dados de eficácia ou segurança de ECRs. Informações de mundo real sobre a persistência a um 
determinado tratamento, por exemplo, poderiam tornar o processo mais efetivo.

\section{Discussão 4 - Criação de modelos para antecipar esforços com base na análise dos dados}

A previsibilidade para a tomada de decisão, possibilitada pela organização dos dados disponíveis, foi abordada também na plenária do congresso. Tal previsibilidade pode se dar por meio da construção de uma matriz de decisão institucional ou por modelos preditivos de risco.

Sobre a existência de matrizes de decisão, a maioria dos representantes de diferentes modelos assistenciais brasileiros relatou não apresentar na sua prática, exceto para aquele que possui assistência com estrutura verticalizada. Os outros perfis de tomadores de decisão participantes do painel relataram não existir um modelo institucionalizado para a tomada de decisão e esse processo ocorre de maneira empírica, variando de acordo com as demandas apresentadas no cotidiano.

Já sobre a utilização de modelos de predição de risco, essa é uma realidade para todos os modelos assistenciais, apesar de ainda se manter majoritariamente no modelo fee for service. Para determinadas condições de saúde, a existência de informações permite que sejam criados pacotes em que os serviços geralmente utilizados são negociados previamente entre prestador de serviço e operadora. Essa previsibilidade, por meio da construção dos pacotes, permite melhor cotação de preços e promove a garantia de fornecimento do serviço dentro do modelo de financiamento atualmente vigente. Além disso, os dados disponíveis auxiliam no acompanhamento desses pacotes de tratamento por intermédio de uma avaliação periódica, podendo ser desfeito quando é observado que o paciente pode estar sendo tratado de maneira inadequada.

\section{Discussão 5 - Realização de parcerias para a construção de dados de mundo real}

Por meio das discussões realizadas, tanto na plenária do congresso quanto com os gestores brasileiros, foi possível entender que os dados de mundo real auxiliam na eficiência da tomada de decisão, ou seja, na definição de uma estratégia no menor tempo possível e com maior assertividade. Nesse contexto, a indústria farmacêutica surge como um possível facilitador do processo de geração de evidências de mundo real por intermédio de diferentes formas de parceria.

Há, atualmente, um interesse da indústria farmacêutica na realização dessas parcerias para a geração de evidências de mundo real, que pode ser observado em diferentes países da América Latina. Esses esforços estão concentrados, principalmente, na geração de dados de maior qualidade, focando em sistemas informatizados. O Brasil é, hoje, um país da América Latina com grande investimento nessas parcerias, não somente pelo seu tamanho, mas também pelas informações disponíveis.

Questionados sobre a possibilidade de realização de parcerias para a geração de evidências de mundo real entre

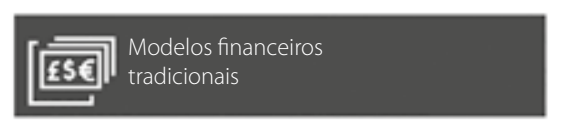

Modelos baseados em preço vs. volume e/ou negociação direta de descontos

\begin{tabular}{l} 
Fatores de implementação \\
Complexidade metodológica \\
e de cálculo \\
Estrutura interna (TI, processos, \\
compliance etc.) \\
Uso de evidências de mundo real \\
\hline
\end{tabular}

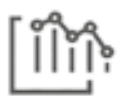

- Desconto linear sob o preço lista

- Definição de deflator sob o preço médio de mercado

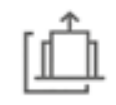

- Contratos de precificação por camadas ou price-cap

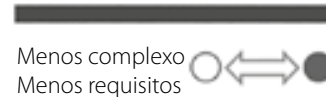

Mais complexo Mais requisitos

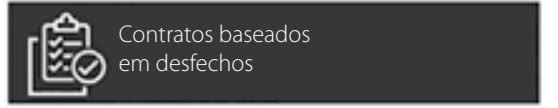

Precificação/descontos não lineares, compartilhamento de risco e contratos baseados em desfechos

\begin{tabular}{l}
\hline \multicolumn{1}{|c|}{ Fatores de implementação } \\
Complexidade metodológica \\
e de cálculo \\
Estrutura interna (TI, processos, \\
compliance etc.) \\
Uso de evidências de mundo real \\
\hline
\end{tabular}

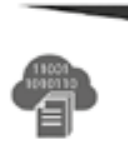

Baseados em desfecho/ compartilhamento de risco (EHR/contas médicas)

- Contratos baseados em eventos clínicos (contas médicas)

- Geração de evidências de mundo real (inclusão condicional)

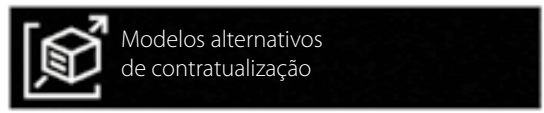

Não tradicionais/Não baseados em desfecho Securitização, Phased Pricing e modelos de assinatura

\begin{tabular}{l} 
Fatores de implementação \\
$\begin{array}{l}\text { Complexidade metodológica } \\
\text { e de cálculo }\end{array}$ \\
$\begin{array}{l}\text { Estrutura interna (Tl, processos, } \\
\text { compliance etc.) }\end{array}$ \\
\hline Uso de evidências de mundo real \\
\hline
\end{tabular}

- Custo de cura (One-Off)

- Pagamento por assinatura

- Pagamentos faseados/parcelas

- Securitização/fundo solidário - Resseguro/Stop-Loss

Figura 1. Modelos de contratualização e partilha de risco.

Fonte: Desenvolvimento próprio adaptado de Agência Nacional de Saúde Suplementar (ANS) (2019), MIT (2019) e Papanicolas et al. (2018) (Agência Nacional de Saúde Suplementar (ANS), 2019b; Massachussetts Institute of Technology, 2019; Papanicolas et al., 2018). 
indústrias e as operadoras de planos de saúde, os gestores relataram que existem ainda barreiras a serem sobrepostas para esse tipo de colaboração. As razões pelas quais esse desafio existe podem estar relacionadas tanto a crenças quanto à influência comercial, imposições de compliance, indisponibilidade de pessoal e tempo para realização de tarefas, entre outras. Porém, existe uma tendência de mudança de paradigma, por meio da troca para novas gestões e realização de parcerias menores como treinamentos para equipe técnica.

\section{Discussão de caso - Utilização de dados de mundo real para a tomada de decisão na perspectiva dos imunobiológicos}

O uso de imunobiológico é um tema recorrente nas discussões das operadoras de saúde devido ao seu impacto epidemiológico e cronicidade dos tratamentos. Dentro desse cenário, os gestores participantes do painel foram convidados a refletir como os dados de mundo real poderiam auxiliar no processo de tomada de decisão, qual seria a limitação para seu uso e como contorná-la.

Os gestores concordaram que dados de mundo real podem gerar um cenário mais fidedigno sobre a utilização de recursos e custos gerados pelos pacientes na prática, e que essa análise poderia levar a um embasamento mais robusto para a tomada de decisão quanto à escolha de imunobiológicos como, por exemplo, o conhecimento sobre o tempo de permanência dos indivíduos em linhas específicas de tratamento, que poderia ser um fator decisório para a escoIha do tratamento. Como principais limitações, destacam-se a ausência de informação e a falta de recursos para análise dos dados, e a transformação deles em evidência passível de uso na tomada de decisão. O maior envolvimento de profissionais no processamento e na análise de dados, além de mudanças em questões regulatórias seriam necessários para a melhoria do processo.

\section{Análise complementar: percepção dos desafios na utilização de dados de mundo real}

Dos vinte questionários enviados, a pesquisa teve participação de 13 gestores (Tabela 1), e a representatividade da amostra foi dividida entre cooperativa (38\%), medicina de grupo (23\%), autogestão (15\%), seguradora (15\%) e autarquia (8\%).
A maior parte dos participantes (66\%) era de operadoras de grande porte, acima de 100 mil beneficiários com estrutura parcialmente verticalizada (50\%).

\section{Utilização de dados de mundo real, finalidades e confiabilidade}

No que tange ao uso de dados mundo real, grande parte da amostra (92\%) reportou já ter utilizado esse tipo de evidência internamente nos últimos três anos. Esse uso se deu especialmente para fins de gerenciamento interno e processos comerciais como o levantamento de custos para análises financeiras e gerenciais (85\%), o levantamento de custos e a utilização de recursos de saúde para a negociação com fornecedores e prestadores (69\%), e para a autorização de cobertura (69\%). Entre respondentes, todos os cinco tipos de operadoras reportaram utilizar evidências de mundo real para esses tipos de processos internos. Por outro lado, em processos de atualização de formulários e protocolos (69\%), estudos econômicos para a cobertura extra rol (46\%) e estudos de mundo real com a perspectiva acadêmica e de publicações (38\%), seguradoras e autarquias não reportaram a utilização. Finalmente, apenas 15\% dos respondentes relataram utilizar evidências de mundo real de modo mais sofisticado em análises estatísticas preditivas e utilizando inteligência artificial (Figura 2).

Apesar de a maior parte dos gestores entrevistados reportar a utilização de evidências de mundo real para tomada de decisão durante o processo de avaliação de cobertura e autorização, existe uma divisão entre os que utilizam com frequência e aqueles que fazem uso apenas em casos específicos. Mais da metade (54\%) dos respondentes reportaram que utilizam sempre ou consideravelmente quando julgam necessário e outros 38\% reportaram que utilizam poucas vezes ou em casos específicos. Apenas $8 \%$ declararam que nunca utilizaram.

De maneira ampla, os gestores confiam nas evidências de mundo real disponíveis (77\%). Destes, 69\% julgam os dados confiáveis e $8 \%$, muito confiáveis. Os demais os classificam como pouco ou nada confiáveis (23\%).

Mesmo que esses dados sejam utilizados pelas operadoras, a coleta e o controle de qualidade dos dados disponíveis ainda são fatores a serem considerados durante o processo

Tabela 1. Perfil das operadoras de saúde representadas na pesquisa online

\begin{tabular}{|c|c|}
\hline Classificação segundo ANS ${ }^{1}$ & Resposta (\%) \\
\hline Seguradora & 15,38 \\
\hline Medicina de grupo & 23,08 \\
\hline Cooperativa médica & 38,46 \\
\hline Autogestão & 15,38 \\
\hline Autarquia & 7,69 \\
\hline Filantropia & 0,00 \\
\hline
\end{tabular}

${ }^{1}$ ANS - Agência Nacional de Saúde Suplementar. 
de tomada de decisão. A principal razão para tal é o grau de completude dos dados disponíveis, seguida por problemas no processo de input das informações na fonte, e, finalmente, pelo tamanho da amostra disponível referente ao universo da operadora. O histórico dos dados disponíveis foi o fator com menor relevância reportado pelos respondentes. Esses achados estão apresentados na Figura 3 e na Tabela 2.

\section{Disponibilidade, formato e processos na utilização de evidências de mundo real}

Apesar de a utilização das evidências de mundo real ser bastante difundida no SSS, a disponibilidade, formatos e processos para a utilização são bastante variáveis. A realidade das operadoras mostrou-se contrastante, retratando que ou há disponibilidade de dados ou estes são completamente res-

Utilização de dados de mundo real: Processos utilizados por classificação de operadoras

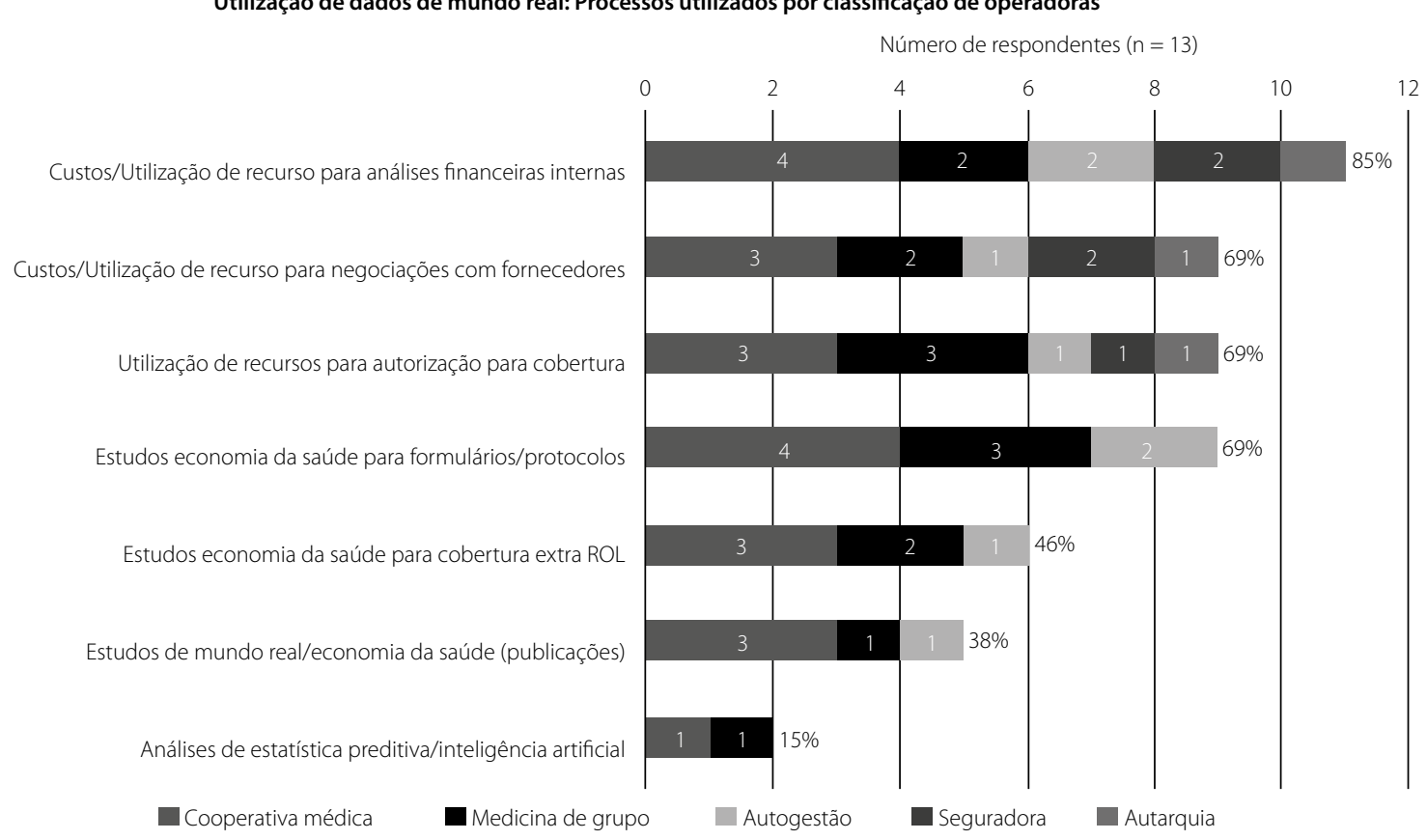

Figura 2. Utilização de dados de mundo real de acordo com o relato dos gestores.

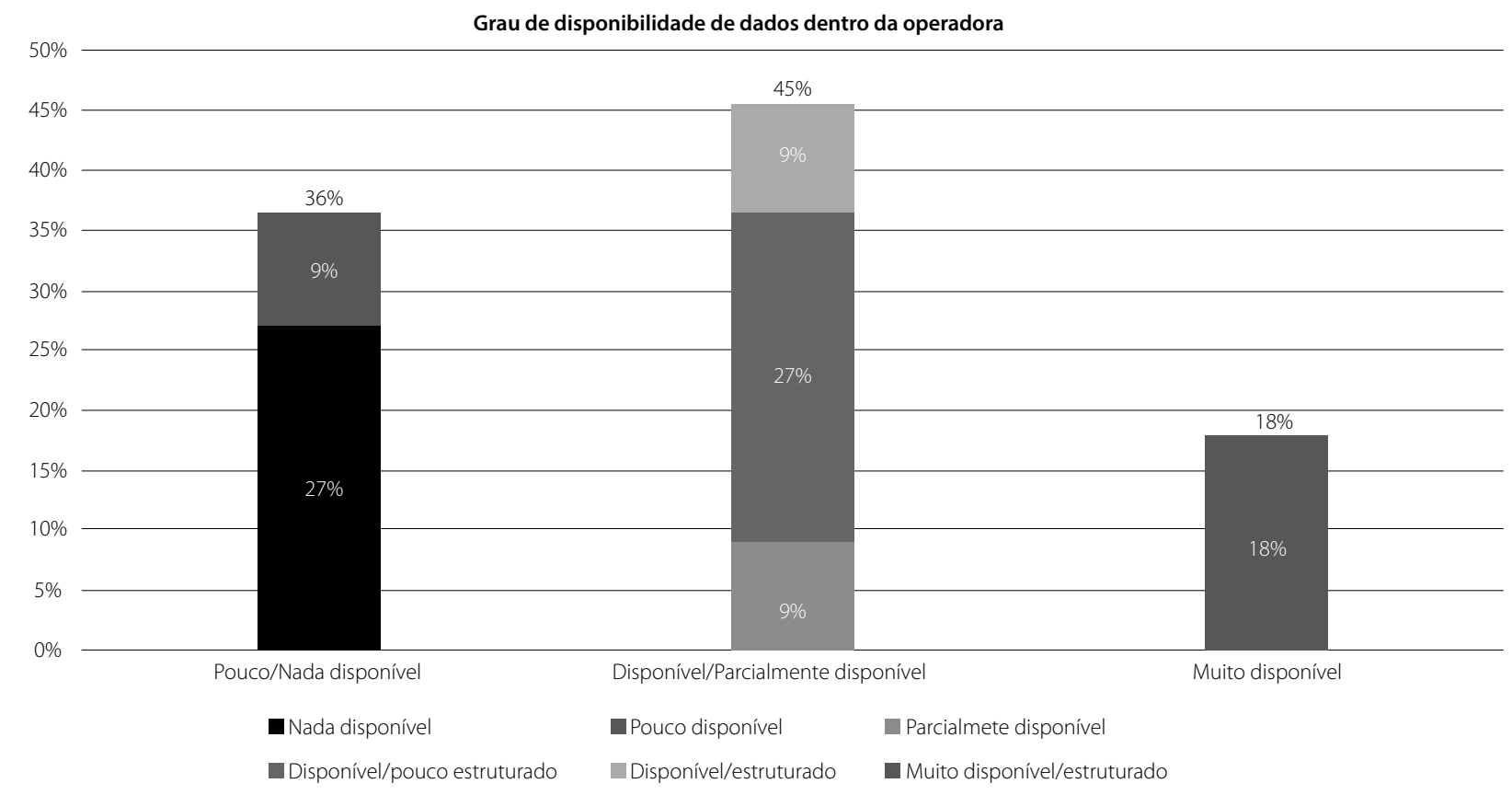

Figura 3. Influência dos critérios no grau de confiabilidade de dados de mundo real. 
tritos. Apenas 10\% consideram os dados muito disponíveis (Figura 4).

Da mesma forma, o formato de acesso aos dados é um fator importante, uma vez que tem influência direta na qualidade da informação disponível e sua granularidade. Dentre os respondentes à pesquisa, grande parte reportou ter acesso a dados de contas médicas (91\%), entretanto, apenas 36\% têm acesso ao conteúdo detalhado desses dados. Ademais, 73\% reportaram ter acesso a prontuários eletrônicos dos pacientes, entretanto, apenas 27\% têm acesso ao conteúdo detalhado dos prontuários, uma vez que para esse tipo de acesso é necessário existir uma integração entre o prestador e o provedor.

\section{Desafios na utilização de dados de mundo real}

Em consonância com as respostas anteriores, o maior desafio encontrado por gestores ainda paira na estrutura de tecnologia/engenharia de dados, seguido pela disponibilidade das evidências e posteriormente por temas relacionados a disponibilidade e capacitação de equipes dedicadas à análise desses dados. A existência de processos estabelecidos e de aprovação da utilização de dados de mundo real foi reportada com menos ênfase, não porque sua importância e impacto são menos relevantes, mas sim porque o resultado dessa análise mostra que, mesmo sendo um tema de amplitude dentro das operadoras, ainda existem temas estruturais a serem endereçados no que tange à utilização de dados de mundo real para suportar a tomada de decisão (Figura 5).

Quanto ao incentivo à diretoria, verificou-se correlação com o perfil da operadora. Para autarquias e autogestões, foi o fator mais relevante, entretanto, para seguradoras e medicinas de grupo, foi o menos relevante. Isso pode estar relacionado ao tamanho e ao grau de estruturação dos processos internos das operadoras.

\section{Discussão}

Diversos fatores têm direcionado mudanças na perspectiva dos gestores, no que tange ao processo de tomada de decisão e o uso de evidências. Dados oriundos de ECRs apresentam diversas limitações e, por isso, a utilização de dados de mundo real vem sendo discutida (Concato et al., 2000). Neste

Tabela 2. Número de respostas de acordo com o grau de importância de cada fator para a confiabilidade nos dados de mundo real

\begin{tabular}{lcccc}
\hline & Grau de completude & Problemas no input & Tamanho da amostra & Histórico dos dados \\
\hline Primeiro fator & 4 & 5 & 3 & - \\
\hline Segundo fator & 5 & 2 & 2 & 3 \\
\hline Terceiro fator & 2 & 2 & 5 & 3 \\
\hline Quarto fator & 1 & 3 & 2 & 6 \\
\hline Média ponderada & 3,00 & 2,75 & 2,50 & 1,75 \\
\hline
\end{tabular}

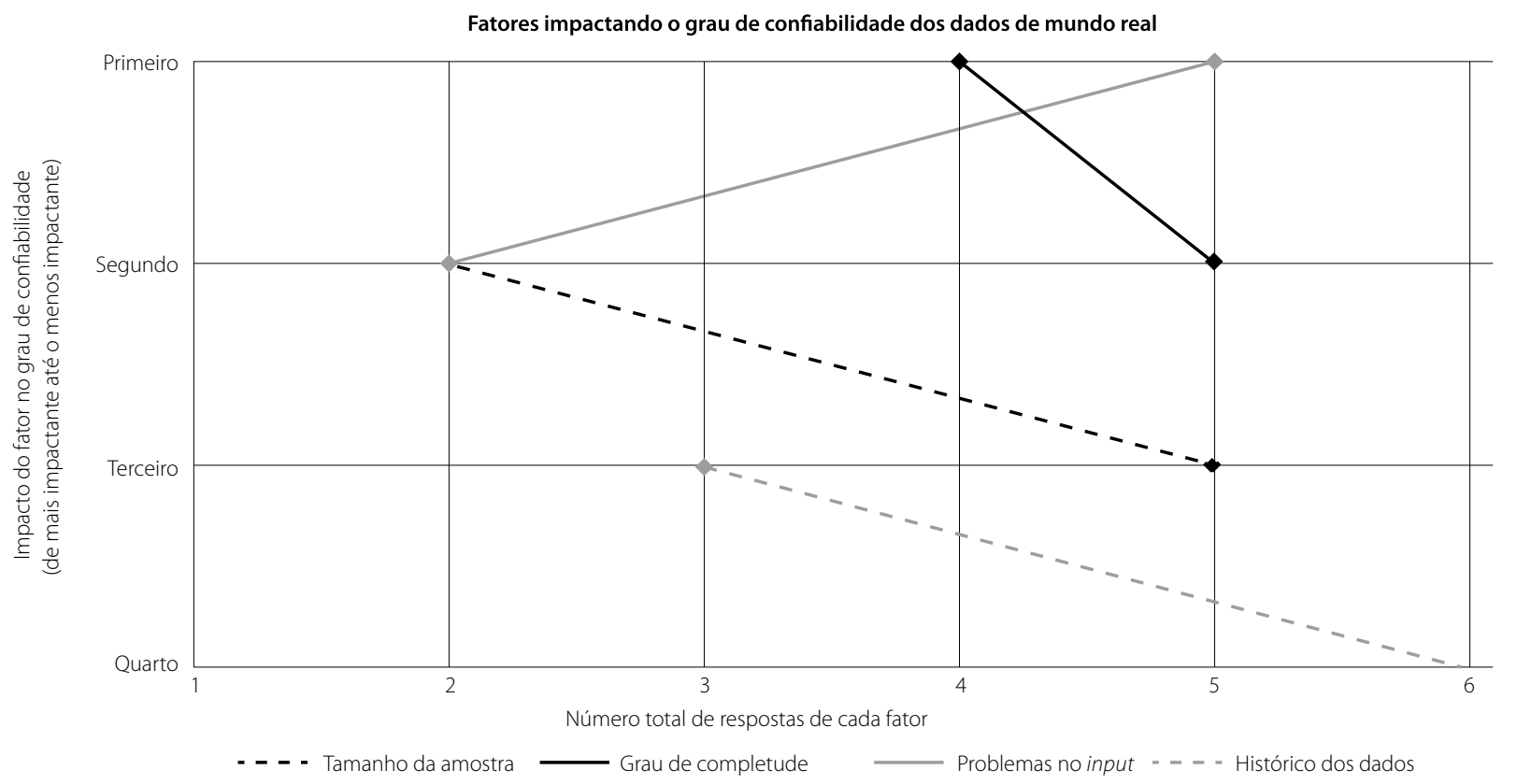

Figura 4. Grau de disponibilidade dos dados nas operadoras de acordo com o relato dos gestores. 
trabalho, foram apresentadas as percepções de diferentes representantes do SSS brasileiro sobre esse tema.

Os resultados do presente estudo reforçam que, atualmente, a tomada de decisão é essencialmente baseada em parâmetros relacionados ao custo do tratamento. No entanto, as discussões demonstraram uma tendência de mudança de paradigma nesse processo, evidenciando a necessidade de atenção a outros aspectos relacionados ao cuidado. Corroborando esse direcionamento, Folter et al. (2018) analisaram os fatores relacionados à tomada de decisão da agência inglesa (National Institute of Health and Care Excellence - NICE) e descrevem características predominantes nesse processo: custo-efetividade; eficácia clínica; características dos estudos disponíveis sobre a tecnologia; características da condição clínica; características da prática atual; necessidades clínicas; tecnologia classificada como inovadora; entre outros (Folter et al., 2018). À vista disso, é possível afirmar que considerar apenas a variável custo, fora da realidade da prática assistencial das operadoras de planos de saúde, pode levar a decisões equivocadas pela existência de outras questões que devem ser consideradas. Sendo assim, o uso de dados de mundo real, que também podem ser obtidos por meio de bases administrativas, pode auxiliar nesse processo, sendo um exemplo a criação de indicadores para acompanhamento de performance das tecnologias reembolsadas.

Os dados reportados neste estudo demonstram também que, apesar do interesse em implementar essa mudança no processo de tomada de decisão, existem algumas dificuldades relacionadas à incorporação deste à rotina de trabalho, pois sua consolidação exige não só uma preparação prévia da organização como também (i) infraestrutura de tecnologia, (ii) treinamento e capacitação das equipes, e (iii) engajamento do corpo diretivo da operadora. Nesse sentido, para transpor alguns desses desafios, uma alternativa possível é a adoção de variáveis simples, de fácil acompanhamento e que já sejam coletadas nas bases existentes, mesmo essas sendo administrativas. Ademais, outra estratégia importante para alavancar essa mudança de paradigma no setor é o incentivo à publicação das análises feitas com o intuito de estimular o intercâmbio de informações e permitir benchmarks.

Somado ao contexto, em uma reflexão mais ampla, verifica-se ainda que, para a implementação de modelos mais modernos baseados em valor, os quais vêm sendo fomentados pela ANS, há uma correlação dos desafios; questões como a ausência de sistemas de informação, falta de dados para estimar o potencial de economias e riscos envolvidos e o pouco conhecimento do perfil epidemiológico e demográfico das populações assistidas, com informações relacionadas aos riscos populacionais e sua distribuição geográfica, são apresentados como um possível empecilho para essa implementação (Agência Nacional de Saúde Suplementar (ANS), 2019c). Por isso, apesar da crescente discussão no cenário nacional sobre o tema, o modelo de financiamento baseado no pagamento por procedimento (feefor service) ainda é predominante, correspondendo a cerca de $96 \%$ dos valores pagos a prestadores de serviço no ano de 2018.

Em vista disso, a ANS instituiu grupos de trabalho para fomentar a discussão de novas estratégias para a tomada de decisão que abrangessem outros modelos e suas possibilidades de implementação no SSS. O resultado desse movimento foi concretizado na publicação do Guia para Implementação de Modelos de Remuneração Baseado em Valor, em janeiro de 2019. Sumarizando as características apontadas pela referida diretriz, é possível observar a importância do tema, no qual características como o acompanhamento de resultados do tratamento e não somente o custo passam a determinar a

Grau de importância dos desafios na utilização de dados de mundo real (1 mais importante/6 menos importante)

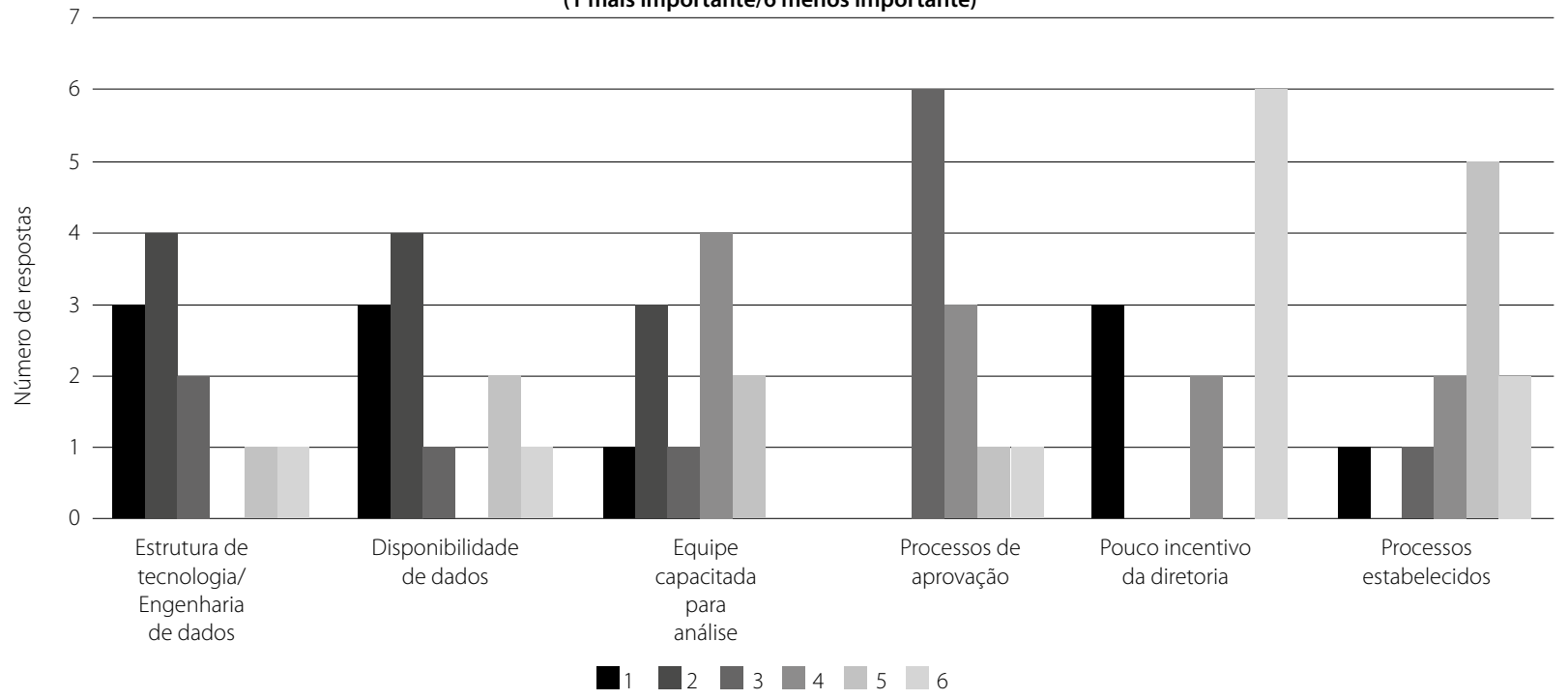

Figura 5. Grau de relevância dos desafios para utilização de dados de mundo real nas operadoras de saúde. 
tomada de decisão (Agência Nacional de Saúde Suplementar (ANS), 2019c):

- Promover a troca de informações entre os envolvidos e a transparência dos dados;

- Promover os estímulos corretos: remuneração mais adequada ao contexto e utilização de protocolos e diretrizes clínicas baseadas em evidências;

- Medir, analisar, relatar e comparar os resultados rigorosamente;

- Alinhar interesses entre os envolvidos: responsabilidade compartilhada;

- Acompanhar desfechos e custos dos pacientes;

- Promover a coordenação do cuidado com enfoque na centralidade no paciente;

- Evitar desperdício no sistema;

- Entender que não existe um modelo único de saúde baseada em valor que se adapte a todas as situações e instituições;

- Focar na melhoria da saúde, e não na cura de doenças.

Da mesma forma, uma comissão interna da ANS discutiu ao longo dos anos de 2016 e 2017 o compartilhamento de risco entre operadoras e modelos de securitização, resultando na publicação da normativa 430/2017, que regulamentou o compartilhamento da gestão de riscos na saúde suplementar (Agência Nacional de Saúde Suplementar (ANS), 2017).

Além da mudança de paradigma quanto aos modelos de financiamento, a ANS tem proposto a implementação de programas cujo objetivo é acompanhar estratégias de promoção de saúde, visando ao cuidado integral e não apenas ao tratamento da doença, como o PROMOPREV (Promoção da Saúde e Prevenção de Riscos e Doenças) e o QUALISS (Programa de Qualificação dos Prestadores de Serviços de Saúde) (Agência Nacional de Saúde Suplementar (ANS), 2019d; Organização Pan-Americana da Saúde (OPAS), 2019). A utilização de dados de mundo real é também capaz de auxiliar nessa transição do modelo de cuidado, uma vez que possibilita a identificação de necessidades existentes na população assistida, além de auxiliar no monitoramento desses programas.

Outra proposta atual em que a disponibilidade de dados de mundo real pode auxiliar refere-se ao acompanhamento da qualidade do serviço de prestadores de saúde (Agência Nacional de Saúde Suplementar (ANS), 2019e). Ao longo do painel de especialistas, a dificuldade de regulação dos serviços prestados foi um ponto levantado pelos gestores participantes. A utilização de dados de mundo real foi citada como capaz de auxiliar nessa avaliação. Sendo assim, entende-se que, a partir da estruturação de dados na operadora, uma discussão com base na razoabilidade das informações entre agência regulatória, prestador e operadora é possível.

\section{Conclusão}

O uso de dados de mundo real se mostra relevante para a tomada de decisão sob a perspectiva do SSS brasileiro, sendo esse tipo de análise considerada um caminho a ser buscado por diferentes estratégias. Ao longo da discussão, foi possível a identificação de mitos e verdades dentro desse processo.

A necessidade de um sistema de dados com tecnologia avançada pode parecer um grande desafio para o processo. Porém, percebeu-se que não é necessário esse tipo de ferramenta para iniciar a utilização dos dados e que, quanto mais simples for esse processo, mais assertiva será a decisão tomada. Outro mito é o de que não existem recursos disponíveis para a utilização de dados de mundo real. Durante o painel, foi possível constatar que a tomada de decisão baseada nesse tipo de informação já é realizada na prática, apesar de nem sempre se ter consciência sobre esse uso. Existe, também, a crença de que a análise dos dados e a tomada de decisão devem ser um processo institucional, porém o acompanhamento de variáveis simples que são coletadas já pode suportar algumas discussões. Além disso, foi notado que existem regras que parecem ser criadas a partir de crenças individuais e que são perpetuadas ao longo do tempo, mas que podem ser quebradas por mudanças de paradigmas.

Dessa forma, percebeu-se que as operadoras brasileiras estão avançadas no tema enxergando o uso de evidências de mundo real como uma tendência iminente para amparar as decisões em saúde. Como qualquer inovação e mudança de paradigma, ainda há muitas dificuldades estruturais a serem enfrentadas, apesar disso, essa estratégia já é percebida como uma alternativa para superar os desafios do setor.

\section{Conflitos de interesse}

André Ballalai prestou serviços de consultoria para indústria farmacêutica. Tassia Decimoni e Camila Rufino são funcionárias da Janssen-Cilag Farmacêutica. Nadja Rehem, Janay Silva, Marina Suavina e Cláudia Souza declaram não haver conflitos de interesse.

\section{Agradecimentos}

Agradecemos à SENSE Company pelo apoio com a redação médica durante o desenvolvimento do esboço deste manuscrito. Este apoio foi financiado pela Janssen.

\section{Referências}

Agência Nacional de Saúde Suplementar. Sala de Situação - ANS - Agência Nacional de Saúde Suplementar [site na Internet]. 2019a [cited on Sept 30 2019]. Available from: http://www.ans.gov.br/perfil-do-setor/dados-eindicadores-do-setor/sala-de-situacao.

Agência Nacional de Saúde Suplementar (ANS). ANS TABNET - Informações em saúde suplementar [site na Internet]. 2019b [cited on Oct 4 2019]. Available from: http://www.ans.gov.br/anstabnet/cgi-bin/tabnet?dados/ tabnet_br.def.

Agência Nacional de Saúde Suplementar (ANS). Guia para Implementação de Modelos de Remuneração baseados em valor. 2019c. p. 1-110. 
Agência Nacional de Saúde Suplementar (ANS). Promoção da Saúde e Prevenção de Riscos e Doenças (PROMOPREV) [site na Internet]. 2019d [cited on Oct 25 2019]. Available from: http://www.ans.gov.br/planos-de-saude-e-operadoras/espaco-da-operadora/compromissos-e-interacoes-com-ans/programas-ans/promoprev-programa-de-promocao-da-saude-e-prevencao-de-riscos-e-doencas.

Agência Nacional de Saúde Suplementar (ANS). Programa de Qualificação dos Prestadores de Serviços de Saúde (QUALISS) [site na Internet]. 2019e [cited on Oct 25 2019]. Available from: http://www.ans.gov.br/prestadores/qualiss-programa-de-qualificacao-dos-prestadores-de-servicos-de-saude.

Agência Nacional de Saúde Suplementar (ANS). Resolução Normativa - RN No 430, de 7 de dezembro de 2017 [site na Internet]. 2017 [cited on Oct 25 2019]. Available from: http://www.ans.gov.br/component/legislacao/?view=legislacao\&task=TextoLei\&format=raw\&id=MzUyMQ==.

Akhras KS, Alsheikh-Ali AA, Kabbani S. Use of real-world evidence for healthcare decision-making in the Middle East: practical considerations and future directions. Expert Rev Pharmacoeconomics Outcomes Res. 2019;19(3):245-50.

Annas GJ. HIPAA Regulations - A New Era of Medical-Record Privacy? N Engl J Med. 2003 Apr 10;348(15):1486-90.

Concato J, Shah N, Horwitz RI. Randomized, Controlled Trials, Observational Studies, and the Hierarchy of Research Designs. N Engl J Med. 2000 Jun 22;342(25):1887-92.

European Union. Data protection in the EU [site na Internet]. 2018 [cited on Oct 7 2019]. Available from: https://ec.europa.eu/info/law/law-topic/ data-protection/data-protection-eu.

Folter J de, Trusheim M, Jonsson P, Garner S. Decision-components of NICE's technology appraisals assessment framework. Int J Technol Assess Health Care. 2018;34(2):163-71.

Franklin JM, Glynn RJ, Martin D, Schneeweiss S. Evaluating the Use of Nonrandomized Real-World Data Analyses for Regulatory Decision Making. Clin Pharmacol Ther. 2019;105(4):867-77.

Franklin JM, Schneeweiss S. When and How Can Real World Data Analyses Substitute for Randomized Controlled Trials? Clin Pharmacol Ther. 2017;102(6):924-33.
Hampson G, Towse A, Dreitlein WB, Henshall C, Pearson SD. Real-world evidence for coverage decisions: Opportunities and challenges. J Comp Eff Res. 2018;7(12):1133-43.

Husereau D, Nason E, Ahuja T, Nikaï E, Tsakonas E, Jacobs P. Use of Real-World Data Sources for Canadian Drug Pricing and Reimbursement Decisions: Stakeholder Views and Lessons for Other Countries. Int J Technol Assess Health Care. 2019;35(3):181-8.

Justo N, Espinoza MA, Ratto B, Nicholson M, Rosselli D, Ovcinnikova O, et al. Real-World Evidence in Healthcare Decision Making: Global Trends and Case Studies From Latin America. Value Heal. 2019;22(6):739-49.

Katkade VB, Sanders KN, Zou KH. Real world data: An opportunity to supplement existing evidence for the use of long-established medicines in health care decision making. J Multidiscip Healthc. 2018;11:295-304.

Kim H-S, Lee S, Kim JH. Real-world Evidence versus Randomized Controlled Trial: Clinical Research Based on Electronic Medical Records. J Korean Med Sci. 2018;33(34):e213.

Makady A, de Boer A, Hillege H, Klungel O, Goettsch W. What Is Real-World Data? A Review of Definitions Based on Literature and Stakeholder Interviews. Value Heal. 2017;20(7):858-65.

Malone DC, Brown M, Hurwitz JT, Peters L, Graff JS. Real-World Evidence: Useful in the Real World of US Payer Decision Making? How? When? And What Studies? Value Heal. 2018;21(3):326-33.

Massachussetts Institute of Technology. Precision Financing Solutions for Durable/Potentially Curative Therapies. FoCUS. 2019.

Organização Pan-Americana da Saúde (OPAS). Folha informativa - Atenção primária à saúde [site na Internet]. 2019 [cited on Oct 11 2019]. Available from: https://www.paho.org/bra/index.php?option=com_content\&view=article\&id=5858:folha-informativa-atencao-primaria-de-saude\& 1 temid $=843$.

Papanicolas I, Woskie LR, Jha AK. Health care spending in the United States and other high-income countries. JAMA. 2018;319(10):1024-39.

Sherman RE, Anderson SA, Dal Pan GJ, Gray GW, Gross T, Hunter NL, et al. Real-world evidence - What is it and what can it tell us? N Engl J Med. 2016;375(23):2293-7. 
